\title{
Natural transformations of higher order cotangent bundle functors
}

\author{
by JAN KUREK (Lublin)
}

\begin{abstract}
We determine all natural transformations of the $r$ th order cotangent bundle functor $T^{r *}$ into $T^{s *}$ in the following cases: $r=s, r<s, r>s$. We deduce that all natural transformations of $T^{r *}$ into itself form an $r$-parameter family linearly generated by the $p$ th power transformations with $p=1, \ldots, r$.
\end{abstract}

Using general methods developed in [2]-[5], we deduce that all natural transformations of the $r$ th order cotangent bundle functor $T^{r *}$ into itself form an $r$-parameter family generated by the $p$ th power transformations $A_{p}^{r, r}$ with $p=1, \ldots, r$.

Then we deduce that all natural transformations of $T^{r *}$ into $T^{(r+q) *}$ form an $r$-parameter family generated by the generalized $p$ th power transformations $A_{p}^{r, r+q}$ with $p=q+1, \ldots, q+r$.

Moreover, we deduce that all natural transformations of $T^{r *}$ into $T^{(r-q) *}$ form an $(r-q)$-parameter family generated by the generalized $p$ th power transformations $A_{p}^{r, r-q}$ with $p=1, \ldots, r-q$.

The author is grateful to Professor I. Kolář for suggesting the problem and for valuable remarks and useful discussions.

1. Let $M$ be a smooth $n$-dimensional manifold. Let $T^{r *} M=J^{r}(M, \mathbb{R})_{0}$ be the space of all $r$-jets $j_{x}^{r} f$ of smooth functions $f: M \rightarrow \mathbb{R}$ with source at $x \in M$ and target at $0 \in \mathbb{R}$. The fibre bundle $\pi_{M}: T^{r *} M \rightarrow M$ with source $r$-jet projection $\pi_{M}: j_{x}^{r} f \mapsto x$ has a canonical structure of a vector bundle with

$$
j_{x}^{r} f+j_{x}^{r} g=j_{x}^{r}(f+g), \quad k \cdot j_{x}^{r} f=j_{x}^{r}(k \cdot f)
$$

for $x \in M$ and $k \in \mathbb{R}[1]$. 
The vector bundle $\pi_{M}: T^{r *} M \rightarrow M$ is called the $r$-th cotangent bundle over $M$

Every local diffeomorphism $\varphi: M \rightarrow N$ is extended to a vector bundle morphism $T^{r *} \varphi: T^{r *} M \rightarrow T^{r *} N, j_{x}^{r} f \mapsto j_{\varphi(x)}^{r}\left(f \circ \varphi^{-1}\right)$, where $\varphi^{-1}$ is locally defined. Hence, the $r$ th cotangent bundle functor $T^{r *}$ is defined on the category $\mathcal{M} f_{n}$ of smooth $n$-dimensional manifolds with local diffeomorphisms as morphisms and has values in the category $\mathcal{V B}$ of vector bundles.

If $\left(x^{i}\right)$ are local coordinates on $M$, then we have the induced fibre coordinates $\left(u_{i}, u_{i_{1} i_{2}}, \ldots, u_{i_{1} \ldots i_{r}}\right)$ on $T^{r *} M$ (symmetric in all indices):

$$
\begin{gathered}
u_{i}\left(j_{x}^{r} f\right)=\left.\frac{\partial f}{\partial x^{i}}\right|_{(x)}, \quad u_{i_{1} i_{2}}\left(j_{x}^{r} f\right)=\left.\frac{\partial^{2} f}{\partial x^{i_{1}} \partial x^{i_{2}}}\right|_{(x)}, \ldots, \\
u_{i_{1} \ldots i_{r}}\left(j_{x}^{r} f\right)=\left.\frac{\partial^{r} f}{\partial x^{i_{1}} \ldots \partial x^{i_{r}}}\right|_{(x)} .
\end{gathered}
$$

Since the functor $T^{r *}$ takes values in the category $\mathcal{V B}$ of vector bundles, we may define natural transformations $A_{p}^{r, r}$ of $T^{r *}$ into itself for $p=1, \ldots, r$.

Definition 1. The natural transformation $A_{p}^{r, r}$ of the $r$ th cotangent bundle functor $T^{r *}$ into itself defined by

$$
A_{p}^{r, r}: j_{x}^{r} f \mapsto j_{x}^{r}(f)^{p},
$$

where $(f)^{p}$ denotes the $p$ th power of $f$, is called the $p$-th power transformation.

Definition 2. The natural transformation $A_{p}^{r, s}$ of $T^{r *}$ into $T^{s *}$ defined by

$$
A_{p}^{r, s}: j_{x}^{r} f \mapsto j_{x}^{s}(f)^{p}
$$

is called the generalized $p$-th power transformation.

We note that in the case $s=r+q$ this definition is correct only for $p=q+1, \ldots, q+r$.

Definition 3. The natural transformation $P^{r, r-q}$ of $T^{r *}$ into $T^{(r-q) *}$ defined by

$$
P^{r, r-q}: j_{x}^{r} f \mapsto j_{x}^{r-q} f
$$

is called a projection.

Note that

$$
A_{p}^{r, r-q}=A_{p}^{r-q, r-q} \circ P^{r, r-q} \quad \text { for } p=1, \ldots, r-q .
$$

2. In this part we determine, by induction on $r$, all natural transformations of $T^{r *}$ into itself. 
ThEOREM 1. All natural transformations $A: T^{r *} \rightarrow T^{r *}$ form the $r$ parameter family

$$
A=k_{1} A_{1}^{r, r}+k_{2} A_{2}^{r, r}+\ldots+k_{r} A_{r}^{r, r}
$$

with any real parameters $k_{1}, k_{2}, \ldots, k_{r} \in \mathbb{R}$.

Proof. The functor $T^{r *}$ is defined on the category $\mathcal{M} f_{n}$ of $n$-dimensional smooth manifolds with local diffeomorphisms as morphisms and is of order $r$. Thus, its standard fibre $S=\left(T^{r *} \mathbb{R}^{n}\right)_{0}$ is a $G_{n}^{r}$-space, where $G_{n}^{r}$ is the group of all invertible $r$-jets from $\mathbb{R}^{n}$ into $\mathbb{R}^{n}$ with source and target at 0 .

According to general standard methods [2]-[5], the natural transformations $A: T^{r *} \rightarrow T^{r *}$ are in bijection with the $G_{n}^{r}$-equivariant maps $f^{r, r}:\left(T^{r *} \mathbb{R}^{n}\right)_{0} \rightarrow\left(T^{r *} \mathbb{R}^{n}\right)_{0}$ of the standard fibres.

Let $\widetilde{a}=a^{-1}$ denote the inverse element in $G_{n}^{r}$ and let

$$
t_{\left(i_{1} \ldots i_{r}\right)}=\frac{1}{r !} \sum_{\sigma \in S_{r}} t_{i_{\sigma(1)} \ldots i_{\sigma(r)}}
$$

denote the symmetrization of a tensor with components $t_{i_{1} \ldots i_{r}}$.

By (1.2) the action of an element $\left(a_{j_{1}}^{i}, a_{j_{1} j_{2}}^{i}, \ldots, a_{j_{1} \ldots j_{r}}^{i}\right) \in G_{n}^{r}$ on $\left(u_{i_{1}}, u_{i_{1} i_{2}}, \ldots, u_{i_{1} \ldots i_{r}}\right) \in\left(T^{r *} \mathbb{R}^{n}\right)_{0}$ is given by

$$
\begin{aligned}
& \bar{u}_{i_{1}}=u_{j_{1}} \widetilde{a}_{i_{1}}^{j_{1}}, \quad \bar{u}_{i_{1} i_{2}}=u_{j_{1} j_{2}} \widetilde{a}_{i_{1}}^{j_{1}} \widetilde{a}_{i_{2}}^{j_{2}}+u_{j_{1}} \widetilde{a}_{i_{1} i_{2}}^{j_{1}}, \ldots, \\
& \bar{u}_{i_{1} \ldots i_{r}}=u_{j_{1} \ldots j_{r}} \widetilde{a}_{i_{1}}^{j_{1}} \ldots \widetilde{a}_{i_{r}}^{j_{r}}+u_{j_{1} \ldots j_{r-1}} \frac{r !}{(r-2) ! 2 !} \widetilde{a}_{\left(i_{1}\right.}^{j_{1}} \ldots \widetilde{a}_{i_{r-2}}^{j_{r-2}} \widetilde{a}_{\left.i_{r-1} i_{r}\right)}^{j_{r-1}}+\ldots \\
& \quad+u_{j_{1} \ldots j_{r-s}}\left[\frac{r !}{(r-s-1) !(s+1) !} \widetilde{a}_{\left(i_{1}\right.}^{j_{1}} \ldots \widetilde{a}_{i_{r-s-1}}^{j_{r-s-1}} \widetilde{a}_{\left.i_{r-s} \ldots i_{r}\right)}^{j r-s}+\ldots\right] \\
& \quad+u_{j_{1} j_{2}}\left[\frac{r !}{(r-1) ! 1 !} \widetilde{a}_{\left(i_{1}\right.}^{j_{1}} \widetilde{a}_{\left.i_{2} \ldots i_{r}\right)}^{j_{2}}+\ldots\right]+u_{j_{1}} \widetilde{a}_{i_{1} \ldots i_{r}}^{j_{1}} .
\end{aligned}
$$

I. Consider the case $r=2$. The equivariance of a $G_{n}^{2}$-equivariant map $f^{2,2}=\left(f_{i}, f_{i j}\right)$ of $\left(T^{2 *} \mathbb{R}^{n}\right)_{0}$ into itself with respect to homotheties in $G_{n}^{2}$ : $\widetilde{a}_{j}^{i}=k \delta_{j}^{i}, \widetilde{a}_{j_{1} j_{2}}^{i}=0$, gives the homogeneity conditions

$$
k f_{i}\left(u_{i}, u_{i j}\right)=f_{i}\left(k u_{i}, k^{2} u_{i j}\right), \quad k^{2} f_{i j}\left(u_{i}, u_{i j}\right)=f_{i j}\left(k u_{i}, k^{2} u_{i j}\right) .
$$

By the homogeneous function theorem [2]-[5], we deduce that, first, the $f_{i}$ are linear in $u_{i}$ and independent of $u_{i j}$, and secondly, the $f_{i j}$ are linear in $u_{i j}$ and quadratic in $u_{i}$. Using the invariant tensor theorem for $G_{n}^{1}[2]-[5]$, we obtain $f^{2,2}$ in the form

$$
f_{i}=k_{1} u_{i}, \quad f_{i j}=k_{2} u_{i} u_{j}+k_{3} u_{i j}
$$

with any real parameters $k_{1}, k_{2}, k_{3} \in \mathbb{R}$. 
The equivariance of $f^{2,2}$ of the form (2.5) with respect to the kernel of the projection $G_{n}^{2} \rightarrow G_{n}^{1}: \widetilde{a}_{j}^{i}=\delta_{j}^{i}$ and $\widetilde{a}_{j k}^{i}$ arbitrary, gives

$$
k_{3}=k_{1} \text {. }
$$

This proves our theorem for $r=2$.

II. Suppose that the theorem holds for $r-1$ and the $G_{n}^{r-1}$-equivariant maps $f^{r-1, r-1}$ of $\left(T^{(r-1) *} \mathbb{R}^{n}\right)_{0}$ into itself define the $(r-1)$-parameter family $\bar{A}=k_{1} A_{1}^{r-1, r-1}+\ldots+k_{r-1} A_{r-1}^{r-1, r-1}$ with any real parameters $k_{1}, \ldots, k_{r-1}$ $\in \mathbb{R}$.

Our aim is to obtain the general form of any $G_{n}^{r}$-equivariant map of $\left(T^{r *} \mathbb{R}^{n}\right)_{0}$ into itself.

Let $\left(u_{1}, u_{2}, \ldots, u_{r}\right):=\left(u_{i_{1}}, u_{i_{1} i_{2}}, \ldots, u_{i_{1} \ldots i_{r}}\right)$ denote the fibre coordinates on $T^{r *} M$. We assume that a $G_{n}^{r}$-equivariant map $f^{r, r}$ is of the general form $f^{r, r}=\left(f_{1}, \ldots, f_{r-1}, f_{r}\right)$ and the given map $f^{r-1, r-1}$ defines the first $r-1$ components $\left(f_{1}, \ldots, f_{r-1}\right)$ of $f^{r, r}$.

Considering the equivariance of $f^{r, r}$ with respect to the homotheties $\widetilde{a}_{j}^{i}=k \delta_{j}^{i}, \widetilde{a}_{j_{1} j_{2}}^{i}=0, \ldots, \widetilde{a}_{j_{1} \ldots j_{r}}^{i}=0$ in $G_{n}^{r}$, for the $r$ th component $f_{r}$ we obtain the homogeneity condition

$$
k^{r} f_{r}\left(u_{1}, u_{2}, \ldots, u_{r}\right)=f_{r}\left(k u_{1}, k^{2} u_{2}, \ldots, k^{r} u_{r}\right) .
$$

By the homogeneous function theorem [2]-[5], $f_{r}$ is of the general form

$$
\begin{aligned}
f_{i_{1} \ldots i_{r}}= & h_{r} u_{i_{1}} \cdot \ldots \cdot u_{i_{r}}+h_{r-1} u_{\left(i_{1}\right.} \ldots u_{i_{r-2}} u_{\left.i_{r-1} i_{r}\right)} \\
& +\ldots+h_{2,1} u_{\left(i_{1}\right.} u_{\left.i_{2} \ldots i_{r}\right)}+h_{2,2} u_{\left(i_{1} i_{2}\right.} u_{\left.i_{3} \ldots i_{r}\right)}+\ldots+h_{1} u_{i_{1} \ldots i_{r}}
\end{aligned}
$$

with any real parameters $h_{1}, h_{2,1}, h_{2,2}, \ldots, h_{r-1}, h_{r} \in \mathbb{R}$. The equivariance of $f^{r, r}$ with respect to the kernel of the projection $G_{n}^{r} \rightarrow G_{n}^{r-1}: \widetilde{a}_{j}^{i}=\delta_{j}^{i}$, $\widetilde{a}_{j_{1} j_{2}}^{i}=0, \ldots, \widetilde{a}_{j_{1} \ldots j_{r-1}}^{i}=0$ and $\widetilde{a}_{j_{1} \ldots j_{r}}^{i}$ arbitrary, gives

$$
h_{1}=k_{1} \text {. }
$$

Thus, we obtain the 1st power transformation $A_{1}^{r, r}$.

Now, considering the equivariance of $A-k_{1} A_{1}^{r, r}$ with respect to the kernel of the projection $G_{n}^{r-1} \rightarrow G_{n}^{1}: \widetilde{a}_{j}^{i}=\delta_{j}^{i}$ and $\widetilde{a}_{j_{1} j_{2}}^{i}, \ldots, \widetilde{a}_{j_{1} \ldots j_{r-1}}^{i}$ arbitrary, we obtain

$$
h_{2,1}=\frac{r !}{1 !(r-1) !} k_{2}, \quad h_{2,2}=\frac{r !}{2 !(r-2) !} k_{2}, \quad \ldots
$$

Thus, we obtain the 2nd power transformation $A_{2}^{r, r}$.

Then, considering the equivariance of $A-k_{1} A_{1}^{r, r}-k_{2} A_{2}^{r, r}$ with respect to the kernel of the projection $G_{n}^{r-2} \rightarrow G_{n}^{1}$, we obtain the general form of the $3 \mathrm{rd}$ power transformation $A_{3}^{r, r}$. Continuing this procedure gives the next power transformations $A_{3}^{r, r}, \ldots, A_{r-2}^{r, r}$. The equivariance of $A-k_{1} A_{1}^{r, r}-$ $k_{2} A_{2}^{r, r}-\ldots-k_{r-2} A_{r-2}^{r, r}$ with respect to the kernel of the projection $G_{n}^{2} \rightarrow G_{n}^{1}$ : 
$\widetilde{a}_{j}^{i}=\delta_{j}^{i}$ and $\widetilde{a}_{j k}^{i}$ arbitrary, leads to the next relation

$$
h_{r-1}=\frac{r !}{(r-2) ! 2 !} k_{r-1} .
$$

Thus, we obtain the $(r-1)$ th power transformation $A_{r-1}^{r, r}$.

Finally, the $G_{n}^{r}$-equivariant map

$$
A-k_{1} A_{1}^{r, r}-k_{2} A_{2}^{r, r}-\ldots-k_{r-1} A_{r-1}^{r, r}=h_{r} A_{r}^{r, r}
$$

is defined by the $r$ th power transformation with any real parameter $h_{r} \in \mathbb{R}$. If we put $h_{r}=k_{r}$, this proves our theorem.

3. In this part we determine all natural transformations $T^{r *} \rightarrow T^{s *}$ in two cases: $r<s$ and $r>s$.

THEOREM 2. All natural transformations $A: T^{r *} \rightarrow T^{(r+q) *}$ form the $r$-parameter family

$$
A=k_{q+1} A_{q+1}^{r, r+q}+k_{q+2} A_{q+2}^{r, r+q}+\ldots+k_{q+r} A_{q+r}^{r, r+q}
$$

with any real parameters $k_{q+1}, k_{q+2}, \ldots, k_{q+r} \in \mathbb{R}$.

P r o of. We apply induction on $q$.

I. Consider the case $q=1$. According to general standard methods [2][5], the natural transformations $A: T^{r *} \rightarrow T^{(r+1) *}$ are in bijection with the $G_{n}^{r+1}$-equivariant maps of the standard fibres $f^{r, r+1}:\left(T^{r *} \mathbb{R}^{n}\right)_{0} \rightarrow$ $\left(T^{(r+1) *} \mathbb{R}^{n}\right)_{0}$.

Considering the equivariance of $f^{r, r+1}=\left(f_{1}, \ldots, f_{r}, f_{r+1}\right)$ with respect to homotheties: $\widetilde{a}_{j}^{i}=k \delta_{j}^{i}, \widetilde{a}_{j_{1} j_{2}}^{i}=0, \ldots, \widetilde{a}_{j_{1} \ldots j_{r+1}}^{i}=0$, we obtain the homogeneity conditions

$$
\begin{aligned}
& k f_{1}\left(u_{1}, u_{2}, \ldots, u_{r}\right)=f_{1}\left(k u_{1}, k^{2} u_{2}, \ldots, k^{r} u_{r}\right), \ldots, \\
& k^{r} f_{r}\left(u_{1}, u_{2}, \ldots, u_{r}\right)=f_{r}\left(k u_{1}, k^{2} u_{2}, \ldots, k^{r} u_{r}\right), \\
& k^{r+1} f_{r+1}\left(u_{1}, u_{2}, \ldots, u_{r}\right)=f_{r+1}\left(k u_{1}, k^{2} u_{2}, \ldots, k^{r} u_{r}\right) .
\end{aligned}
$$

Additionally, using the equivariance of $f^{r, r}=\left(f_{1}, \ldots, f_{r}\right)$ with respect to the kernel of the projection $G_{n}^{r} \rightarrow G_{n}^{1}$, we obtain, by Theorem 1 , the $r$ parameter family of the form (2.1): $\bar{A}=k_{1} A_{1}^{r, r}+k_{2} A_{2}^{r, r}+\ldots+k_{r} A_{r}^{r, r}$ with any real parameters $k_{1}, k_{2}, \ldots, k_{r} \in \mathbb{R}$.

Moreover, by the homogeneous function theorem and the invariant tensor theorem [2]-[5], we deduce that the $(r+1)$ th component $f_{r+1}$ is of the general form

$$
\begin{aligned}
f_{i_{1} \ldots i_{r+1}}= & l_{r+1} u_{i_{1}} u_{i_{2}} \ldots u_{i_{r+1}}+l_{r} u_{\left(i_{1}\right.} \ldots u_{i_{r-1}} u_{\left.i_{r} i_{r+1}\right)} \\
& +\ldots+l_{2,1} u_{\left(i_{1}\right.} u_{\left.i_{2} \ldots i_{r+1}\right)}+l_{2,2} u_{\left(i_{1} i_{2}\right.} u_{\left.i_{3} \ldots i_{r+1}\right)}+\ldots
\end{aligned}
$$

with any real parameters $l_{2,1}, l_{2,2}, \ldots, l_{r}, l_{r+1} \in \mathbb{R}$. 
The equivariance of $f^{r, r+1}$ with respect to the kernel of the projections $G_{n}^{r+1} \rightarrow G_{n}^{r}$ and $G_{n}^{r+1} \rightarrow G_{n}^{1}$ gives the relations

$$
\begin{aligned}
k_{1} & =0, \\
l_{2,1}=\frac{(r+1) !}{r ! 1 !} k_{2}, \quad l_{2,2} & =\frac{(r+1) !}{(r-1) ! 2 !} k_{2}, \ldots, l_{r}=\frac{(r+1) !}{(r-1) ! 2 !} k_{r} .
\end{aligned}
$$

If we put $l_{r+1}=k_{r+1}$, this gives the $r$-parameter family $f^{r, r+1}=$ $\left(f^{r, r}, f_{r+1}\right)$ of the form

$$
A=k_{2} A_{2}^{r, r+1}+\ldots+k_{r+1} A_{r+1}^{r, r+1}
$$

with any real parameters $k_{2}, \ldots, k_{r+1} \in \mathbb{R}$.

II. Suppose that the theorem holds for $q-1$ and the $G_{n}^{r+q-1}$-equivariant maps $f^{r, r+q-1}:\left(T^{r *} \mathbb{R}^{n}\right)_{0} \rightarrow\left(T^{(r+q-1) *} \mathbb{R}^{n}\right)_{0}$ define the $r$-parameter family

$$
\bar{A}=k_{q} A_{q}^{r, r+q-1}+k_{q+1} A_{q+1}^{r, r+q-1}+\ldots+k_{q+r-1} A_{q+r-1}^{r, r+q-1}
$$

with any real parameters $k_{q}, k_{q+1}, \ldots, k_{q+r-1} \in \mathbb{R}$.

Consider a $G_{n}^{r+q}$-equivariant map $f^{r, r+q}:\left(T^{r *} \mathbb{R}^{n}\right)_{0} \rightarrow\left(T^{(r+q) *} \mathbb{R}^{n}\right)_{0}$ of the form $f^{r, r+q}=\left(f^{r, r+q-1}, f_{r+q}\right)$.

The equivariance of $f^{r, r+q}$ with respect to the homotheties in $G_{n}^{r+q}$ : $\widetilde{a}_{j}^{i}=k \delta_{j}^{i}, \widetilde{a}_{j_{1} j_{2}}^{i}=0, \ldots, \widetilde{a}_{j_{1} \ldots j_{r+q}}^{i}=0$, gives for the $(r+q)$ th component $f_{r+q}$ the homogeneity condition

$$
k^{r+q} f_{r+q}\left(u_{1}, u_{2}, \ldots, u_{r}\right)=f_{r+q}\left(k u_{1}, k^{2} u_{2}, \ldots, k^{r} u_{r}\right) .
$$

By the homogeneous function theorem and the invariant tensor theorem $[2]-[5], f_{r+q}$ is of the form

$$
\begin{aligned}
f_{i_{1} \ldots i_{r+q}}= & l_{r+q} u_{i_{1}} \ldots u_{i_{r+q}}+l_{r+q-1} u_{\left(i_{1}\right.} \ldots u_{i_{r+q-2}} u_{\left.i_{r+q-1} i_{r+q}\right)} \\
& +\ldots+l_{q+1} u_{\left(i_{1}\right.} \ldots u_{i_{q}} u_{\left.i_{q+1} \ldots i_{q+r}\right)}
\end{aligned}
$$

with any real parameters $l_{q+1}, \ldots, l_{q+r-1}, l_{q+r} \in \mathbb{R}$.

The equivariance of $f^{r, r+q}$ with respect to the kernel of the projections $G_{n}^{r+q} \rightarrow G_{n}^{r}$ and $G_{n}^{r+q} \rightarrow G_{n}^{1}$ gives the relations

$$
\begin{gathered}
k_{q}=0, \ldots \\
l_{q+1}=\frac{(q+r) !}{r ! q !} k_{q+1}, \ldots, l_{q+r-1}=\frac{(q+r) !}{(q+r-2) ! 2 !} k_{q+r-1} .
\end{gathered}
$$

If we put $l_{q+r}=k_{q+r}$, this proves our theorem.

ThEOREM 3. All natural transformations $A: T^{r *} \rightarrow T^{(r-q) *}$ form the $(r-q)$-parameter family

$$
A=k_{1} A_{1}^{r, r-q}+k_{2} A_{2}^{r, r-q}+\ldots+k_{r-q} A_{r-q}^{r, r-q}
$$

with any real parameters $k_{1}, k_{2}, \ldots, k_{r-q} \in \mathbb{R}$.

Pr o of. Applying the same general procedure, we conclude that each $A$ : $T^{r *} \rightarrow T^{(r-q) *}$ is the composition of the projection $P^{r, r-q}: T^{r *} \rightarrow T^{(r-q) *}$ 
and a transformation $\bar{A}$ of $T^{(r-q) *}$ into itself: $A=\bar{A} \circ P^{r, r-q}$. This proves our theorem.

\section{References}

[1] J. Gancarzewicz, Differential Geometry, PWN, Warszawa 1987 (in Polish).

[2] I. Kolár̆, Some natural operators in differential geometry, in: Proc. Conf. Diff. Geom. and its Applications, Brno 1986, Kluwer, Dordrecht 1987, 91-110.

[3] I. Kolář and G. Vosmanska, Natural transformations of higher order tangent bundles and jet spaces, Časopis Pěst. Mat. 114 (2) (1989), 181-186.

[4] I. Kolář, P. Michor and J. Slovak, Natural Operations in Differential Geometry, to appear.

[5] J. Kurek, On natural operators on sectorform fields, Časopis Pěst. Mat. 115 (3) (1990), 232-239.

INSTITUTE OF MATHEMATICS

MARIA CURIE-SKLODOWSKA UNIVERSITY

PL. M. CURIE-SKLODOWSKIEJ 1

20-031 LUBLIN, POLAND 